\title{
A new reference database for beta-delayed neutrons
}

Paraskevi Dimitriou ${ }^{1, *}$, Iris Dillmann², Balraj Singh $^{3}$, Vladimir Piksaikin ${ }^{4}$, Jose-Luis Tain ${ }^{5}$, Krzysztof Rykaczewski ${ }^{6}$, Alejandro Algora ${ }^{5}$, Kaushik Banerjee ${ }^{7}$, Ivan Borzov ${ }^{8}$, Daniel Cano-Ott ${ }^{9}$, Satoshi Chiba ${ }^{10}$, Muriel Fallot ${ }^{11}$, Daniela Foligno $^{12}$, Robert Grzywacz ${ }^{13}$, Xiaolong Huang ${ }^{14}$, Tomislav Marketin ${ }^{15}$, Futoshi Minato ${ }^{16}$, Gopal Mukherjee ${ }^{7}$, BertisCharlie Rasco ${ }^{6}$, Alejandro Sonzogni ${ }^{17}$, and Marco Verpelli ${ }^{1}$

${ }^{1}$ Nuclear Data Section, Division of Physical and Chemical Sciences, International Atomic Energy Agency, Wagramerstrasse 5, A-1400, Vienna, Austria

${ }^{2}$ TRIUMF, Vancouver, British Columbia V6T 2A3, Canada

${ }^{3}$ Department of Physics and Astronomy, McMaster University, Hamilton, Ontario L8S 4M1, Canada

${ }^{4}$ State Scientific Center of Russian Federation, Institute of Physics and Power Engineering, 249033 Obninsk, Russian Federation

${ }^{5}$ IFIC, CSIC-Universitat de Valencia, 46071 Valencia, Spain

${ }^{6}$ Physics Division, Oak Ridge National Laboratory, Oak Ridge, Tennessee 37831, USA

${ }^{7}$ Variable Energy Cyclotron Centre, Kolkata 700064, India

${ }^{8}$ National Research Centre Kurchatov Institute, Department of Nuclear Astrophysics, 1 Akademika Kurchatova pl., Moscow 123182, Russian Federation

${ }^{9}$ Centro de Investigaciones Energéticas, Medioambientales y Tecnológicas (CIEMAT), Avenida Complutense 40, Madrid 28040, Spain

${ }^{10}$ Tokyo Institute of Technology, 2-12-1-N1-9, Ookayama, Meguru-ku, Japan

${ }^{11}$ Subatech, CNRS/in2p3, Univ. of Nantes, IMTA, 44307 Nantes, France

${ }^{12}$ CEA, DEN, DER/SPRC/LEPh Cadarache, F-13108 Saint Paul-Lez-Durance, France

${ }^{13}$ Department of Physics and Astronomy, University of Tennessee, Knoxville, Tennessee 37996, USA

${ }^{14}$ China Nuclear Data Center, China Institute of Atomic Energy, Beijing 102413, China

${ }^{15}$ Department of Physics, Faculty of Science, University of Zagreb, 10000 Zagreb, Croatia

${ }^{16}$ Nuclear Data Center, Nuclear Science and Engineering Center, Japan Atomic Energy Agency, Tokai-mura Ibaraki-ken, Japan

${ }^{17}$ National Nuclear Data Center, Brookhaven National Laboratory, Brookhaven, Upton NY, USA

\begin{abstract}
A new database containing all available experimental and evaluated $\beta$-delayed neutron data is presented in this paper. The database is the product of an international effort coordinated by the International Atomic Energy Agency. It comprises a microscopic section including all available experimental data on betadecay half-lives, $\beta$-delayed neutron emission probabilities and spectra, as well as new systematics and global theoretical calculations for comparison. The beta-delayed neutron data for individual precursors have been benchmarked against available data on macroscopic properties such as total delayed-neutron yields and spectra, delayed-neutron decay curves and time-dependent group parameters using the summation method. All available measured macroscopic data have also been compiled in the macroscopic section of the database. The dedicated database is available online on the IAEA server (URL:http://www-nds.iaea.org/beta-delayedneutron/database.html).
\end{abstract}

\section{Introduction}

Since the first detection of $\beta$-delayed neutrons in 1939 there has been significant effort to understand the mechanism of delayed-neutron emission. In the 70's a large number of measurements of delayed-neutron emission probabilities ( $P_{n}$ values) for fission products (and also for lowmass nuclei in the non-fission region) were performed around nuclear reactors. During the last decade there has been a renewed interest in the experimental and theoretical study of $\beta$-delayed neutron emission at the next generation of radioactive beam facilities mainly focussed on neutronrich nuclei far off the stability line. For this reason, novel

*e-mail: vivian@inp.demokritos.gr. Current address: Institute of Nuclear and Particle Physics, National Centre for Scientific Research "Demokritos", Athens 15341, Greece detectors have been built to operate at these major accelerator facilities to measure the delayed-neutron decay characteristics of $\beta$-delayed neutron-emitters. In parallel, efforts to quantify aggregate properties of delayed neutron emission in fissile materials are ongoing. These efforts are motivated by the need for improved $\beta$-delayed neutron data for a wide range of applications such as calculations of the decay heat produced in reactors, planning of future advance fuel technology, calculating anti-neutrino spectra from reactors, r-process nucleosynthesis, and nuclear structure physics. Although half-lives and $P_{n}$ data are available in several compiled and evaluated libraries such as ENSDF, NUBASE, NuDat, etc., a complete documentation of measurements and evaluation procedures is often missing for these properties. Previous dedicated 
compilations and evaluations also suffer from incompleteness, and have in the meantime become outdated. Measured neutron spectra are not available in any database.

In light of the above, the creation of a modern and comprehensive database where the user could retrieve various types of pertinent information related to beta-delayed neutrons was timely. A Consultants' meeting organized by the IAEA brought together experimentalists, nuclear data evaluators, and theoreticians to explore such a possibility [1]. The recommendations of the report [1] lead to an IAEA Coordinated Research Project (CRP) (20132018) [5] whose objective was to develop a Reference Database for beta-delayed neutrons [2-4] that would cover both microscopic and macroscopic data. The scope of the CRP included: (i) Compilation and evaluation of experimental half-lives and $P_{x n}$ for delayed-neutron precursors, (ii) Establishing standards in different mass regions, for which several independent and reliable measurements are available, (iii) Developing new empirical systematics based on the new evaluated $P_{n}$ data, (iv) Calculating betadecay half-lives and $P_{n}$ values using available empirical and microscopic models, (v) Testing the new evaluated beta-decay half-lives and $P_{n}$ values against macroscopic data and benchmark experiments, (vi) Measuring total delayed neutron yields (TDN) and spectra for a range of actinides, (vii) Re-evaluating 6- and 8-group representations based on new measurements, and (viii) Developing systematics for 6- and 8-group representations.

The results obtained during the IAEA CRP have been described in detail in the final CRP article submitted to Nuclear Data Sheets [6]. In this present paper, we discuss selected outputs of the CRP and present the retrieval interface to the new online database (http://www-nds.iaea.org/ beta-delayed-neutron/database.html).

\section{Microscopic data}

Based on the Atomic Mass Evaluation 2016 (AME2016) [7], 621 out of the 2451 known nuclei are $\beta$-delayed neutron emitters. In 2011, prior to the CRP, only $216 \beta$ delayed neutron emitters had been measured: 81 nuclides in the light mass region $(Z<28), 134$ nuclides in the fission region with $Z=29-57$, and a single nuclide, ${ }^{210} \mathrm{Tl}$ [8], in the mass region with $Z>57$.

At present, $298 \beta 1 \mathrm{n}$-emitters have been measured $\left(P_{1 n}\right.$ values) which constitutes $48 \%$ of all $\beta 1$ n-emitter. For multi-neutron emitters, however, only $23 P_{2 n}$ values $(7.7 \%$ of the total), $4 P_{3 n}$ values (2.9\% of the total), and $1 P_{4 n}$ value $(1.7 \%$ of the total) have been measured. The situation is going to change significantly in the near future, as over 250 new $\beta$-delayed neutron emitters will be measured by the "Beta-delayed neutrons at RIKEN" (BRIKEN) project [9] and data already measured at other facilities such as the RIBBL separator at Lanzhou accelerator facility in China, CERN-ISOLDE, IGISOL, EURICA at RIKEN Nishina Center, Beta-Paul Trap at CARIBU (Argonne National Laboratory) and GRIFFIN at TRIUMF/ISAC, will also become available thus changing the landscape of measured $\beta$-delayed neutron emitters.
For the purposes of the CRP, all experimental data published up until May 2018 were considered. Betadecay half-lives and $\beta$-delayed neutron emission probabilities $P_{n}$ for $649 \beta$-delayed neutron emitters were compiled and evaluated using the standard procedures adopted for the Evaluated Nuclear Structure Data File (ENSDF). The number 649 includes newly discovered neutron-rich nuclei at RIKEN, most of these potential $\beta$-delayed neutron emitters, which are not listed in AME-2016. Of these 649 measured nuclides, 221 were in the lighter mass region $(Z<29)$ and 428 in the fission mass region $(Z>28)$. For each one of these nuclides, the available experimental data and methods were assessed and then the most reliable data were selected for averaging resulting in the recommended value. The evaluated tables have been published in two separate publications covering the lighter mass region $(Z<29)[10]$ and higher fission mass region $(Z>28)$ [11], respectively.

Some of these 649 measured $\beta$-delayed neutron emitters satisfy certain well-defined conditions and can be recommended as 'standards' for $\beta$-delayed neutron $1 \mathrm{n}$ emission: ${ }^{9} \mathrm{Li},{ }^{16} \mathrm{C},{ }^{17} \mathrm{~N},{ }^{49} \mathrm{~K},{ }^{83} \mathrm{Ga},{ }^{87} \mathrm{Br},{ }^{88} \mathrm{Br},{ }^{94} \mathrm{Rb},{ }^{95} \mathrm{Rb},{ }^{137} \mathrm{I}$, ${ }^{145} \mathrm{Cs},{ }^{146} \mathrm{Cs}$, and ${ }^{147} \mathrm{Cs}$. The main criteria for recommending a $\beta$-delayed neutron 'standard' are: a) easy production of large quantities at various facilities using clean beams, b) measured in four or more experiments using reliable methods and c) all measured values are consisten within an uncertainty less than $5 \%$.

New systematics were developed based on the new $\left(T_{1 / 2}, P_{1 n}\right)$ evaluated data using the empirical formulas of Kratz and Herrmann [12], McCutchan et al. [13] and Miernik [14]. While these global systematics show considerable scatter, the corresponding systematics along a given $\mathrm{Z}$ are much more compact and were used to identify outliers that need to be further investigated [11].

The new $\left(T_{1 / 2}, P_{1 n}\right)$ tables were compared systematically with global models of beta-decay, namely with the density functional model coupled to the continuum quasiparticle random-phase approximation (DF+cQRPA) of Borzov [15], the relativistic HartreeBogoliubov+QRPA model (RQRPA) of Marketin et al. [16], and the microscopic-macroscopic finite-range droplet model (FRDM) of Möller et al. [17]. Figure 1 shows a comparison between the three mentioned models and the new evaluated CRP data for Co isotopes.

\section{Macroscopic data}

Recent activities related to macroscopic delayedneutron data have predominantly taken place at IPPE (Obninsk). New measurements of TDNs and of relative abundances of delayed neutrons and half lives of their precursors were performed at IPPE for neutron-induced fission of ${ }^{232} \mathrm{Th},{ }^{233} \mathrm{U},{ }^{235} \mathrm{U},{ }^{236} \mathrm{U},{ }^{238} \mathrm{U},{ }^{239} \mathrm{Pu},{ }^{237} \mathrm{~Np}$, and ${ }^{241} \mathrm{Am}$ in the energy range from thermal to 18 $\mathrm{MeV}$ (see Ref. [6] and references therein). Composite delayed-neutron energy spectra were also measured for thermal neuron-induced fission of ${ }^{235} U$ [18]. All the available experimental data relevant to neutron-induced fission have been compiled in the new macroscopic 


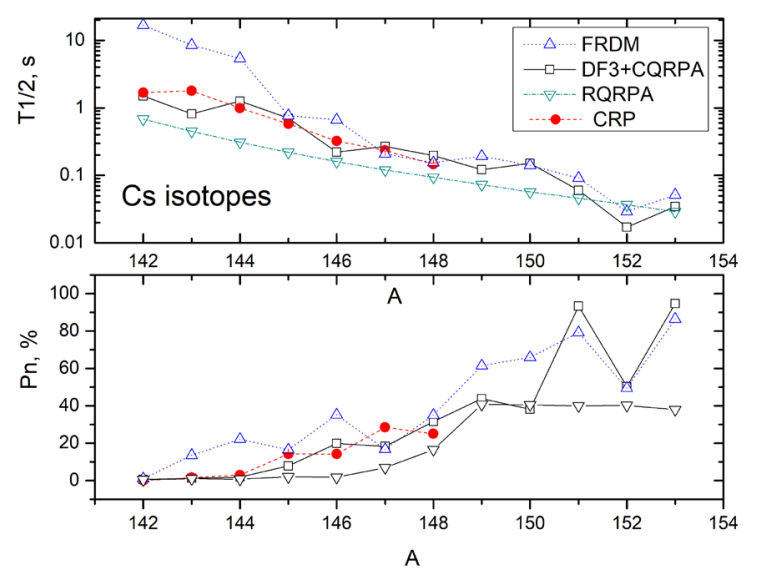

Figure 1. Evaluated $\left(T_{1 / 2}, P_{x n}\right)$ compared with model calculations from DF+cQRPA [15], RQRPA [16] and FRDM [17] for Cs isotopes.

database making it a comprehensive repository of integral delayed-neutron data. The database includes both the compilations of delayed-neutron parameters of Spriggs and Campbell [19] and Tuttle [20] as well as the new IPPE data on TDNs, relative abundances and half-lives in 6- and 8-group models with covariance and correlation data, and composite delayed-neutron energy spectra. Furthermore, new systematics were developed for the time-dependent parameters and new recommendations were made for both 6- and 8-group parameters for thermal, fast and high-energy neutron-induced fission of ${ }^{232} \mathrm{Th},{ }^{233} \mathrm{U},{ }^{235} \mathrm{U}$, ${ }^{236} \mathrm{U},{ }^{238} \mathrm{U},{ }^{237} \mathrm{~Np},{ }^{239} \mathrm{Pu}$ and ${ }^{241} \mathrm{Am}$ actinides. Details can be found in [6] and in references therein.

Macroscopic data were also used to benchmark the newly evaluated $\left(T_{1 / 2}, P_{n}\right)$ data for individual precursors using the summation method. The impact of the new CRP data on the TDNs for fast neutron-induced fission of ${ }^{238} \mathrm{U}$ is shown in Fig. 2. What is clear from the figure is the strong sensitivity of the results to the fission yield data. In fact, it appears that the TDNs are more sensitive to the fission yield data than the $P_{n}$ data. The calculated TDNs agree with the recommended values of Ref. [19] only when JEFF-3.1.1 fission yields are used in the summation calculations. These results stress the need for further investigation into the fission yield libraries.

\section{Beta-delayed neutron reference database}

The compiled experimental data, evaluations, systematics as well as model calculations and recommendations produced by the CRP are included in the reference database which is available online at: http://www-nds.iaea.org/ beta-delayed-neutron/database.html.

The database comprises two sections, the microscopic data and macroscopic data section. In each section the user is able to search per nuclide, energy, and ranges of masses,

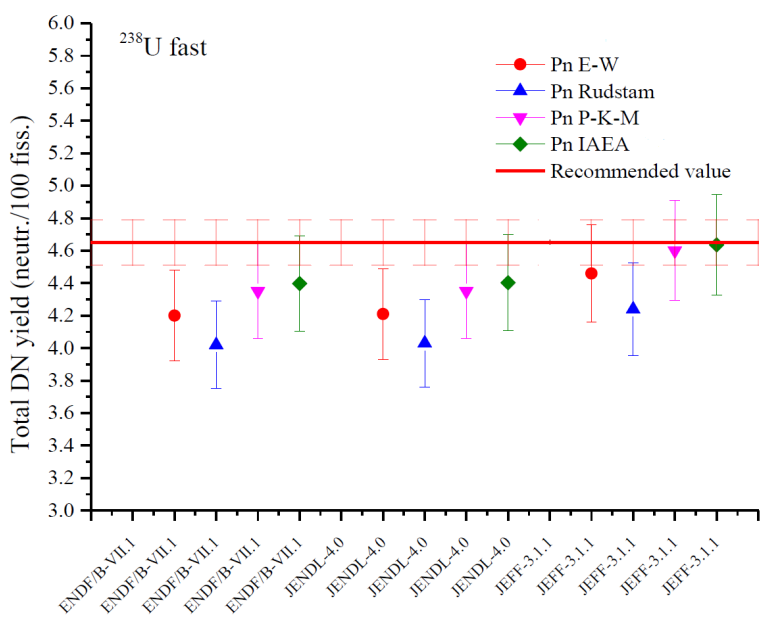

Figure 2. Total delayed-neutron yields calculated using the summation method for fast $n$-induced fission of ${ }^{238} \mathrm{U}$. The different symbols are obtained using different $P_{n}$ compilations: Wilson and England [21] (red circles), Rudstam et al. [22] (blue triangles), Pfeiffer et al. [23] (magenta triangles), CRP data [6] (green diamonds). The fission yield data were obtained from the evaluated libraries ENDF/B-VII.1, JENDL-4.0, and JEFF-3.1.1.

half-lives $T_{1 / 2}$ and $P_{n}$ values. The data can also be downloaded as csv files. A purely numerical table containing the microscopic $\left(T_{1 / 2}, P_{n}\right)$ values has been prepared specifically to be used in summation calculations and can also be downloaded as a csv file.

For the microscopic data, the user is also able to plot the evaluated data and compare them with systematics and model calculations. In the macroscopic section, the user is able to compare the compiled and recommended data for TDNs with the corresponding data in the evaluated libraries. A snapshot of the macroscopic database is shown in Fig. 3.

\section{Conclusions}

The revitalized interest in the measurement of nuclear decay properties of the most neutron-rich nuclei has lead to the development of new detection technologies that have been used in radioactive beam facilities to explore the $\beta$ delayed neutron emission landscape.

The international collaboration coordinated by the IAEA has reviewed both the experimental and theoretical efforts related to the decay of individual $\beta$-delayed neutron emitters that were published until May 2018. Although the full impact of the new $\left(T_{1 / 2}, P_{n}\right)$ data still needs to be explored, it is clear that microscopic theories need to be improved to include deformation, pairing and the competition of Gamow-Teller and First-Forbidden transitions consistently.

The results of summation and time-dependent calculations of TDNs indicate that the higher $P_{1 n}$ branching ratios measured with improved experimental methods for the key $\beta$-delayed neutron emitters in nuclear fuel, such as ${ }^{137} \mathrm{I}$, influence the TDNs, delayed-neutron activity and reactivity. 


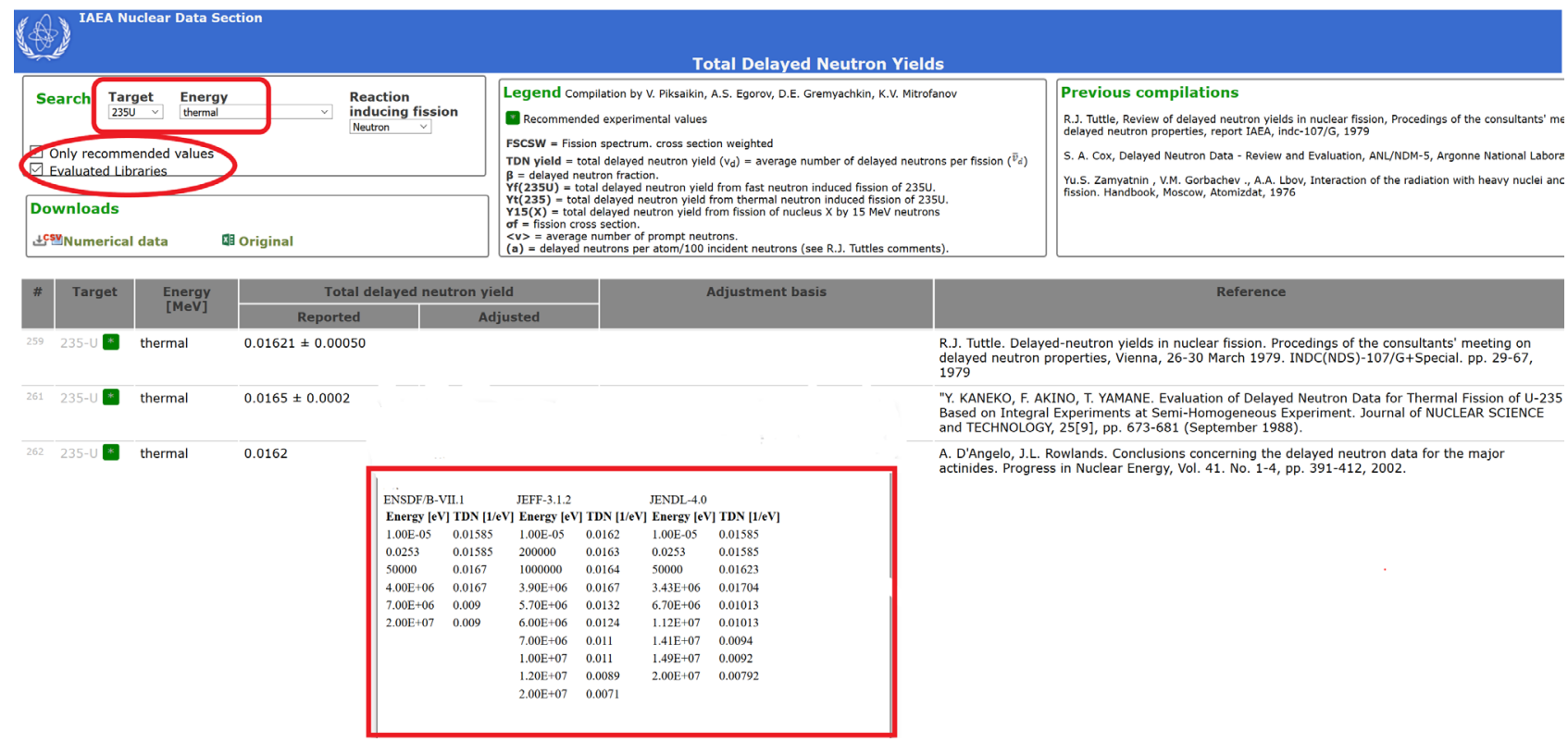

Figure 3. A snapshot of the online Reference Database is shown, where the user can search for experimental total delayed neutron yields for thermal $n$-induced fission of ${ }^{235} \mathrm{U}$ and compare with evaluated libraries.

However, these calculations are rather sensitive to fission yield data and their uncertainties. It is therefore recommended that fission yield data are investigated and updated to reflect the advances in both experimental and theoretical methods. Another important aspect of the summation calculations which however, has not been investigated thorougly as it was beyond the scope of the CRP, is the impact of the uncertainties of both the $P_{x n}$ data and fission yields and their correlations on integral quantities.

The new measurements of integral data for major and minor actinides at a range of incident energies have been used to test the new microsocopic data, develop new systematics for the time-dependent parameters with better predictive power and recommend new 6- and 8-group model parameters. What remains to be done is to verify and validate these new recommended group models.

All the microscopic and and macroscopic data are readily available to the user community at the IAEA online Reference Database (http://www-nds.iaea. org/beta-delayed-neutron/database.html).

\section{References}

[1] D. Abriola, B. Singh, I. Dillmann, Technical Rep. INDC(NDS)-0599, (IAEA, Vienna, 2011)

[2] I. Dillmann, P. Dimitriou, B. Singh, Technical Rep. INDC(NDS)-0643, (IAEA, Vienna, 2014)

[3] I. Dillmann, B. Singh, P. Dimitriou, Technical Rep. INDC(NDS)-0683, (IAEA, Vienna, 2015)

[4] I. Dillmann, P. Dimitriou, B. Singh, Technical Rep. INDC(NDS)-0735, (IAEA, Vienna, 2017)

[5] IAEA CRP website, URL: http://wwwnds.iaea.org/beta-delayed-neutron
[6] P. Dimitriou, I. Dillmann, B. Singh et al., Nuclear Data Sheets, submitted (2019)

[7] G. Audi, F.G. Kondev, M. Wang, W.J. Huang, S. Naimi, Chin. Phys. C 41, 030001 (2017)

[8] G. Stetter, Nucl. Sci. Abstr. 16, Abstr. 10963, 1409 (1962)

[9] A. Tarifeño-Saldivia, J. L. Tain, C. Domingo-Pardo et al., J. Instrumentation 12, P04006 (2017)

[10] M. Birch et al., Nuclear Data Sheets 128, 131-184 (2015)

[11] J. Liang et al., Nuclear Data Sheets, to be published, (2020)

[12] K.-L. Kratz, G. Herrmann, Z. Physik 263, 435 (1973)

[13] E.A. McCutchan et al., Phys. Rev. C 86, 041305(R) (2012)

[14] K. Miernik, Phys. Rev. C 88, 041301(R) (2013)

[15] I.N. Borzov, Phys. Rev. C 71, 065801 (2005)

[16] T. Marketin, L. Huther, G. Martinez-Pinedo, Phys. Rev. C 93, 025805 (2016)

[17] P. Möller, B. Pfeifer, K.-L. Kratz, Phys. Rev. C 67, 055802 (2003)

[18] V.M. Piksaikin, A.S. Egorov, A.A. Goverdovski, D.E. Gremyachkin, K.V. Mitrofanov, Ann. Nucl. Energy 102408 (2017)

[19] G.D. Spriggs and J.M. Campbell, Prog Nucl. Energy 41, 145 (2002)

[20] R.J. Tuttle, Nucl. Sci. Eng. 56, 37 (1975)

[21] W. Wilson, T. England, Prog. Nucl. Energy 41, 71 (2002)

[22] G. Rudstam, K. Aleklett, L. Sihver, At. Data Nucl. Data Tables 53, 1 (1993)

[23] B. Pfeiffer, K.-L. Kratz, P. Möller, Prog. Nucl. Energy 41, 39 (2002) 